\title{
A narrative review of systemic treatment options for hepatocellular carcinoma: state of the art review
}

\author{
Joy Awosika ${ }^{\# \wedge}$, Davendra Sohal ${ }^{\# \wedge}$ \\ Department of Hematology \& Oncology, University of Cincinnati Cancer Center, Cincinnati, OH, USA \\ Contributions: (I) Conception and design: Both authors; (II) Administrative support: None; (III) Provision of study materials or patients: Both authors; \\ (IV) Collection and assembly of data: None; (V) Data analysis and interpretation: None; (VI) Manuscript writing: Both authors; (VII) Final approval \\ of manuscript: Both authors. \\ \#Both authors contributed equally to this work. \\ Correspondence to: Joy Awosika, MD. University of Cincinnati Cancer Center, 234 Goodman Street, 2nd Floor, Cincinnati, Ohio 45219, USA. \\ Email: awosikjy@ucmail.uc.edu.
}

\begin{abstract}
Hepatocellular carcinoma (HCC) is an aggressive cancer that typically develops in the setting of underlying cirrhosis of the liver. HCC commonly presents in advanced stages and if eligible orthotopic liver transplantation (OLT) and surgical resection/ablation remain as the only curative options. Prior to 2007, no systemic therapy was available that demonstrated an improvement in survival. Underlying cirrhosis and poor synthetic hepatic function provides a major challenge into effective systemic options contributing to the poor success of cytotoxic chemotherapy in HCC. The first drug to achieve clinical success was sorafenib despite the underwhelming overall survival of 3 months. Since then, other targeted therapies have shown modest benefit as well. Most recently, immunotherapy advances have come to the forefront in the management of HCC and combination therapy with immunotherapy and monoclonal antibodies have now surpassed sorafenib as first line treatment. This article will review the various approved and emerging therapies that have had a significant clinical impact and highlight the future directions and ongoing research that will hopefully translate into better outcomes in the treatment approach of advanced HCC.
\end{abstract}

Keywords: Immunotherapy; tyrosine kinase inhibitors (TKI); targeted therapy; hepatocellular carcinoma (HCC)

Submitted May 04, 2021. Accepted for publication Dec 14, 2021.

doi: 10.21037/jgo-21-274

View this article at: https://dx.doi.org/10.21037/jgo-21-274

\section{Introduction}

Hepatocellular carcinoma (HCC) accounts for $90 \%$ of primary liver cancers and is the fourth-leading cause of cancer-related deaths worldwide despite multiple treatment modalities that have emerged over the years $(1,2)$. In the United States it is projected that by 2040, HCC will be the third-leading cause of cancer-related death, surpassing breast cancer with an estimated 41,000 deaths per year (3). Incorporation of effective surveillance programs has led to early detection in $40-50 \%$ of HCC making them amenable to early-stage curative therapies such as resection, local ablation, and liver transplantation (4,5). However, despite diligent surveillance programs, a substantial proportion of HCC diagnoses are still made in the advanced stages (6). Non-curative options have consisted of locoregional therapies in intermediate unresectable disease and systemic therapies in advanced disease. Systemic therapies have been driven by the evolution in molecular biology of cancer with the advent of sorafenib in 2007 launching a new era into targeted therapies. Since then, other targeted therapies have been shown to improve clinical outcomes in HCC. More recently, immunotherapy has made considerable progress in HCC treatment. Here, we review systemic therapies,

\footnotetext{
^ ORCID: Joy Awosika, 0000-0001-6074-4771; Davendra Sohal, 0000-0002-0182-8403.
} 
with a focus on recent advances. We present the following article in accordance with the Narrative Review reporting checklist (available at https://jgo.amegroups.com/article/ view/10.21037/jgo-21-274/rc).

\section{Methods}

An online search of the literature utilizing google scholar, PubMed, Cochrane library and science direct was employed. Selection criteria included advanced or unresectable HCC, targeted therapies, immunotherapy, randomized clinical trials from 2007 to April 2021.

\section{Tyrosine kinase inbibitors (TKI)}

Since the discovery of imatinib in 2001, TKIs have rapidly evolved and emerged as approved targeted therapies in various cancers. Protein tyrosine kinase (PTK) is a class of proteins involved in cell signaling and differentiation. The tyrosine kinase activity catalyzes the transfer of phosphate groups on ATP to the tyrosine residues of many important proteins, making proteins phosphorylation, then transferring signal to regulate cell growth, differentiation, death and a series of physiological and biochemical processes (7). Common receptor tyrosine kinases (RTKs) targeted in tumorigenesis are epidermal growth factor receptor (EGFR), platelet-derived growth factor receptor (PDGFR), vascular endothelial growth factor receptor (VEGFR) and insulin receptor (INsR). They typically have an extracellular domain that binds to specific ligands causing a series of biochemical reactions resulting in cellular proliferation (Figure 1). Proto-oncogenes and oncogenes are found to be associated with mutated or abnormal expression of PTKs and play a significant role in cancer growth manifested by tumor invasion and metastasis, tumor neovascularization and tumor chemotherapy resistance (7). This became the main driver behind various research and clinical trials and the discovery of TKIs in the treatment of cancers. It wasn't very long that TKIs in HCC treatment heralded the discovery of sorafenib in 2007 and subsequent various other TKIs that have shown promising outcomes in HCC treatment (Table 1).

\section{Sorafenib}

Prior to 2007 there weren't any effective systemic treatment options available for advanced HCC. The SHARP trial brought to the forefront sorafenib, a multikinase inhibitor that targets VEGF, PDGF and Raf receptors inhibiting tumor- cell proliferation, tumor angiogenesis and increasing the rate of apoptosis (Figure 1). This multicenter, double blinded placebo-controlled trial enrolled 602 patients with advanced HCC who hadn't received prior systemic therapy were assigned to sorafenib $400 \mathrm{mg}$ twice a day or placebo. Primary outcomes were overall survival (OS) and time to symptomatic progression with secondary endpoints of radiological progression and safety. Results from the trial showed a median OS nearly 3 months longer compared with the placebo group [10.7 vs. 7.9 months; hazard ratio $(\mathrm{HR})=0.69 ; \mathrm{P}<0.001]$ (5). With the success of sorafenib and the focus of targeting antiangiogenic pathways in HCC various targeted therapies have since been investigated but failed to produce superiority or noninferiority compared with sorafenib (Table 2). Over the years phase I/II trials have studied dovitinib, vandetanib, pazopanib, orantinib, tivantinib, nintedanib, foretinib in the first-line setting without success.

After about 10 years of sorafenib being the mainstay of systemic therapy in HCC, lenvatinib demonstrated clinical benefit as well in the first-line setting. Lenvatinib is an oral multikinase inhibitor that targets VEGF receptors 1-3, PDGF receptor alpha, FGF receptors, RET and KIT (8). In an open-label, phase III, noninferiority, multicenter clinical trial, 954 eligible patients with unresectable or advanced HCC who had not received treatment for advanced disease were randomly assigned to receive lenvatinib or sorafenib. The primary endpoint of median OS was 13.6 months with lenvatinib compared with 12.3 months with sorafenib meeting criteria for noninferiority (HR $=0.92,95 \%$ CI: $0.72-1.06)(8)$. Secondary end points of median PFS was longer with lenvatinib ( 7.4 months) compared with sorafenib (3.7 months). similarly, a longer time to progression with lenvatinib revealed 8.4 months compared with 3.7 months with sorafenib. Lenvatinib also had an objective response rate (ORR) of $24.1 \%$ compared with $9.2 \%$ on sorafenib (8). Lenvatinib arm had fewer hand-foot-skin reactions, diarrhea, and alopecia events; the sorafenib arm had fewer hypertension, proteinuria, dysphonia, and hypothyroidism events. Overall, the study concluded that lenvatinib was non-inferior to sorafenib as a first-line treatment in advanced HCC.

Lenvatinib and sorafenib are both FDA-approved as first-line therapies in advanced HCC. The decision on which therapy to use is left to the discretion of the physician with lenvatinib demonstrating noninferiority to sorafenib in overall survival per the REFLECT trial (8). Lenvatinib had superior secondary end points of PFS, TTP and RR compared with sorafenib $(8,15)$. Sorafenib has been 


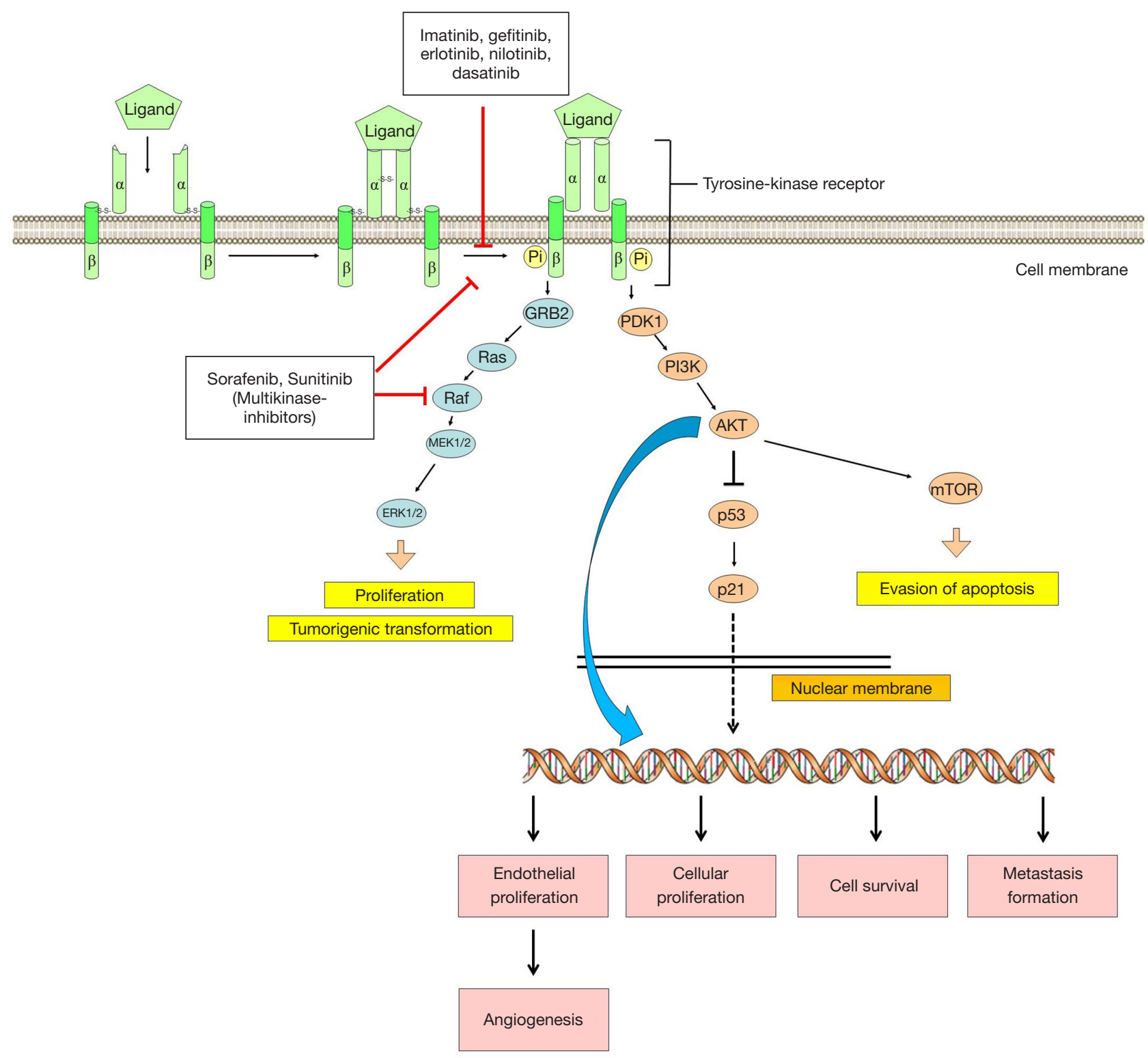

Figure 1 Schematic model of tumorigenic signaling pathways and their inhibition by anti-cancer-TKI. The figure is reused from the article below Eckstein N, Röper L, Haas B, et al. Clinical pharmacology of tyrosine kinase inhibitors becoming generic drugs: the regulatory perspective. J Exp Clin Cancer Res 2014;33:15.

around for over 10 years leading to more experience and familiarity with its use. Safety profile was comparable for the two agents, with lenvatinib better tolerated with regard to diarrhea, weight loss and hand-foot skin reactions and sorafenib causing less hypertension, hypothyroidism, and proteinuria (8). Subsequent clinical trials evaluating secondline therapeutic options have been conducted in the setting of prior sorafenib use and none yet in the setting of prior lenvatinib therapy (15).

\section{Cabozantinib}

In patients who have been previously treated with sorafenib and developed disease progression on at least one systemic therapy, cabozantinib, a multikinase inhibitor, has shown clinical activity and promising outcomes in advanced HCC (9). Cabozantinib targets VEGF, MET and AXL receptors. MET and AXL receptors have been implicated in antiangiogenic resistance in addition to epithelial- 
Table 1 Lists a summary of the TKI's that are FDA approved in advanced HCC

\begin{tabular}{|c|c|c|c|c|c|c|c|}
\hline Trial name & Author & $\begin{array}{c}\text { No. of } \\
\text { patients }\end{array}$ & Study arm & ECOG & $\begin{array}{l}\text { Child-Pugh } \\
\text { score }\end{array}$ & $\begin{array}{l}\text { BCLC } \\
\text { stage }\end{array}$ & Primary endpoint \\
\hline SHARP & $\begin{array}{l}\text { Llovet } \\
\text { et al. (5) }\end{array}$ & 602 & $\begin{array}{l}\text { Sorafenib vs. placebo } \\
\quad \text { (first-line setting) }\end{array}$ & 0 to 2 & $A$ & B & $\begin{array}{l}\text { OS: } 10.7 \text { vs. } 7.9 \text { months; hazard ratio }(\mathrm{HR})=0.69 \text {; } \\
\mathrm{P}<0.001 \text {; TTP: } 4.1 \text { vs. } 4.9 \text { months, } \mathrm{P}=0.77\end{array}$ \\
\hline REFLECT & $\begin{array}{l}\text { Kudo } \\
\text { et al. (8) }\end{array}$ & 954 & $\begin{array}{l}\text { Lenvantinib vs. } \\
\text { sorafenib (first-line } \\
\text { setting) }\end{array}$ & 0 or 1 & $A$ & $\mathrm{~B}$ or $\mathrm{C}$ & $\begin{array}{l}\text { OS: lenvatinib } 13.6 \text { months (95\% Cl: } 12.1-14.9) \text { vs. } \\
\text { sorafenib (12.3 months, } 95 \% \mathrm{Cl}: 10.4-13.9) \\
(\mathrm{HR}=0.92,95 \% \mathrm{Cl}: 0.79-1.06)\end{array}$ \\
\hline RESORCE & $\begin{array}{l}\text { Bruix } \\
\text { et al. (10) }\end{array}$ & 567 & $\begin{array}{l}\text { Regorafenib vs. } \\
\text { placebo (second-line } \\
\text { setting) }\end{array}$ & 0 or 1 & A & $\mathrm{B}$ or $\mathrm{C}$ & $\begin{array}{l}\text { OS: } 10.6 \text { months }(95 \% \mathrm{Cl}: 9.1-12.1) \text { for } \\
\text { regorafenib vs. } 7.8 \text { months }(6.3-8.8) \text { for placebo, } \\
\mathrm{HR}=0.63 \text { (95\% Cl: } 0.50-0.79 ; \mathrm{P}<0.0001)\end{array}$ \\
\hline
\end{tabular}

TKI, tyrosine kinase inhibitors; HCC, hepatocellular carcinoma; OS, overall survival; HR, hazard ratio.

Table 2 lists the main phase III trials that failed to demonstrate noninferiority to sorafenib

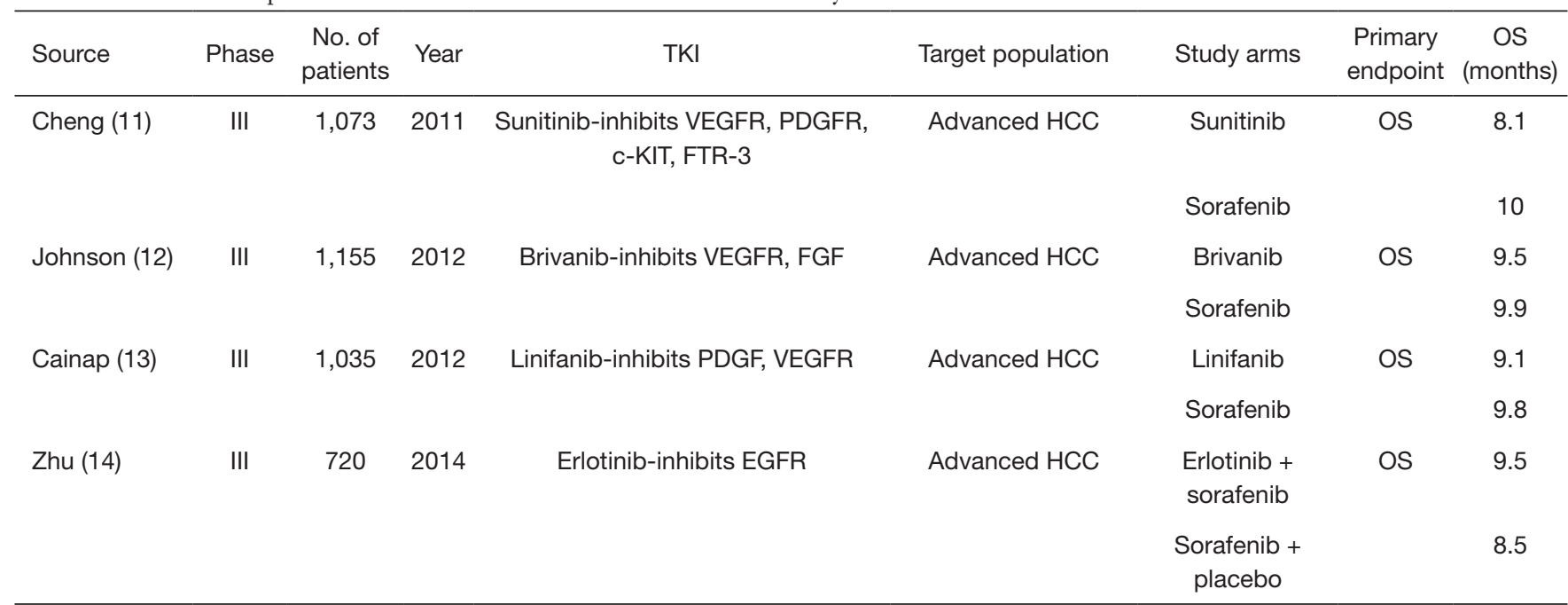

TKI, tyrosine kinase inhibitors; VEGFR, vascular endothelial growth factor receptor; PDGFR, platelet derived growth factor receptor; FGF, fibroblast growth factor; HCC, hepatocellular carcinoma; OS, overall survival.

mesenchymal transition, invasion, and metastasis (9). Poor prognosis in HCC has been associated with high expression of MET and AXL with increased MET activity seen in previously treated patients on sorafenib who develop resistance (9). In this randomized, double-blinded phase III trial, 707 patients who had received prior treatment with sorafenib, had developed progression of disease on at least one systemic treatment for HCC, or received up to two previous systemic treatments, were enrolled to receive cabozantinib $60 \mathrm{mg}$ daily $v s$. placebo (9). The primary endpoint was OS and secondary endpoints were PFS and ORR. Patients receiving cabozantinib had longer OS (10.2 vs. 8.0 months; $\mathrm{HR}=0.76 ; 95 \%$ CI: $0.63-0.92 ; \mathrm{P}=0.005$ ). PFS was 5.2 vs. 1.9 months, and ORR was $4 \%$ and $<1 \%$, comparing cabozantinib and placebo, respectively (9). Grade 3 or 4 adverse events occurred in $68 \%$ of patients receiving cabozantinib with the most common observed being hand-foot skin reactions, hypertension, fatigue, diarrhea and increase in hepatic AST levels (9).

\section{Regorafenib}

The RESORCE trial introduced regorafenib as a potential second-line agent for advanced HCC in patients who had 


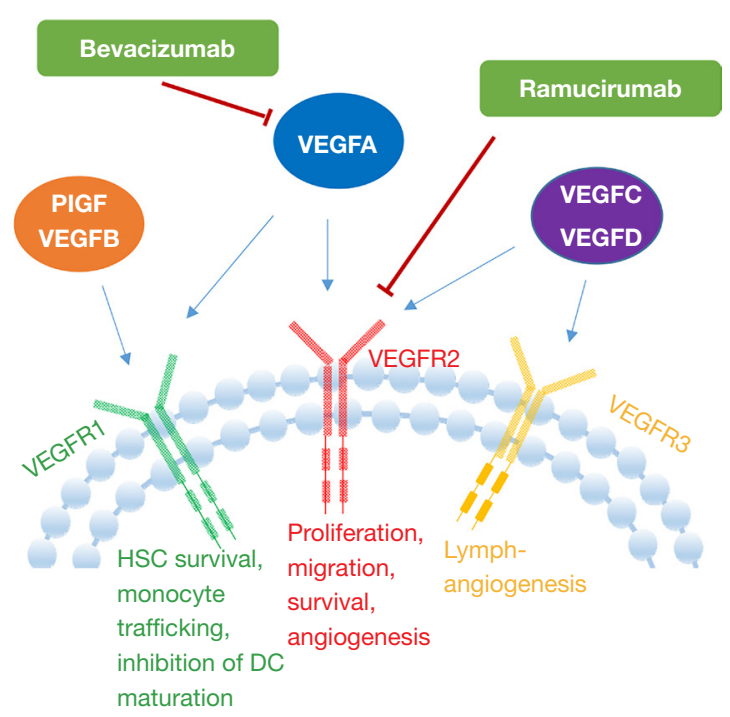

Figure 2 Mechanism of action of anti-vascular endothelial growth factor monoclonal antibodies. The figure is reused from the article below Gao F, Yang C. Anti-VEGF/VEGFR2 Monoclonal Antibodies and their Combinations with PD-1/PD-L1 Inhibitors in Clinic. Curr Cancer Drug Targets 2020;20:3-18.

progressed on sorafenib (10). Regorafenib is a multikinase inhibitor that targets EGFR and VEGF receptors. The phase III, randomized, double-blinded trial selected 567 Child-Pugh A patients who had been on sorafenib and tolerated the drug for at least 20 out of the 28 days before discontinuation and assessed overall survival compared with placebo (10). Patients were enrolled and randomized to receive regorafenib $160 \mathrm{mg}$ daily $v s$. placebo. Median OS was 10.6 months with regorafenib vs. 7.8 months with placebo (HR $=0.63,95 \%$ CI: 0.50-0.79; $\mathrm{P}<0.0001$ ). Grade 3 or 4 adverse events were hypertension, hand-foot skin reactions, fatigue, and diarrhea (10).

\section{VEGF inbibitors}

Insight into the pathogenesis of HCC has revealed the key role of VEGF and VEGFR signaling pathways in angiogenesis and tumor growth. The VEGF family of proteins are essential regulators in physiological and pathological angiogenesis (16). Their role as therapeutic targets in HCC was discovered when VEGF overexpression was evident in these tumors (17). With the understanding that a key step in tumor growth requires oxygen and nutrient delivery for sustained growth, it is of no surprise that as far back as 1971 the hypothesis was launched regarding targeting tumor angiogenesis as a potential therapeutic option (18). In HCC, heightened VEGF expression is seen and sorafenib as mentioned before also targets the VEGF signaling pathway via multikinase inhibition resulting in the therapeutic benefit well described. The modest benefit of sorafenib (relatively 3 months), however, led to the investigation of alternative pathways targeting VEGF inhibition and tumor angiogenesis (19).

\section{Ramucirumab}

Ramucirumab is a recombinant monoclonal IgG1 antibody and VEGF antagonist (17). In the randomized multicenter phase III REACH trial, ramucirumab was assessed as a second-line treatment option versus placebo in advanced HCC in patients whose disease had progressed on sorafenib. Even though the study did not meet its primary endpoint of OS $v s$. placebo (median OS $9.2 v s$. 7.6 months), further analysis demonstrated that ramucirumab seemed to improve OS in patients with a higher baseline alpha-fetoprotein (AFP) level, shifting attention to the utility of AFP level as a potential biomarker in directing therapy. AFP has historically been used in the diagnosis and prognostication of HCC. Elevated AFP has been associated with elevated VEGFR expression, increased angiogenesis, and poor prognosis in HCC $(17,20)$. REACH 2 was designed to investigate the benefit of ramucirumab in patients with a baseline AFP level of $>400 \mathrm{ng} / \mathrm{mL}$. In this randomized, double-blinded phase III trial, 292 patients were randomized to ramucirumab [197] vs. placebo [95]. Primary endpoint of OS was improved: 8.5 vs. 7.3 months $(\mathrm{P}=0.0199)$; secondary endpoints were also better: PFS: 2.8 vs. 1.6 months $(\mathrm{P}<0.0001)$; and disease control rate: $59.9 \%$ vs. $38.9 \%(\mathrm{P}=0.0006)$ compared with placebo (21).

\section{Bevacizumab}

Bevacizumab, a humanized monoclonal antibody that binds VEGF-A is thought to reduce tumor vascularity and growth (Figure 2). Multiple phase II clinical trials have investigated bevacizumab as either monotherapy (22), combination therapy with EGFR inhibitor (23-26) and combination therapy with chemotherapy (capecitabine, oxaliplatin, gemcitabine) (19,27-29). A systematic review that analyzed 8 phase II clinical trials evaluating bevacizumab as single or combination therapy in advanced HCC concluded that bevacizumab may be effective and tolerated. Unfortunately, limitations based on sample size, lack of randomization, and 
publication bias resulted in none of these trials being able to translate into larger, phase III studies (22). Ongoing research led to the evaluation of combination anti-PDL-1 inhibition with bevacizumab which launched the groundbreaking IMBrave150 phase III study, discussed below.

\section{Immunotherapy}

HCC is commonly referred to as an immunogenic tumor due to its development in chronically inflamed livers from both viral and non-viral causes (30). In addition, expression of tumor-associated antigens and specific gene mutations giving rise to private neoantigens contribute to HCC immunogenicity (31). Research into HCC's tumor microenvironment (TME) has opened the door to novel immunomodulation therapeutic options in advanced HCC. An intricate microenvironment exists between the chronic inflammation sustained from hepatitis viruses and the production of cytokines and growth factors within the parenchyma (30). The liver has also been known to have an intrinsic immunosuppressive microenvironment due to weak $\mathrm{T}$ cell activation from low expressions of tumor related antigens. The body's anti-tumor immune activity is mostly provided by $\mathrm{T}$ cells and upregulation of programmed cell death ligand 1 (PD-L1) on tumor cells has contributed to the immuno-suppressive microenvironment (32). Immune checkpoints are coinhibitory surface glycoproteins that primarily play the role of preventing $\mathrm{T}$ cell immune overactivation during states of inflammation and infection. This limits collateral tissue damage in physiological states but their expression is a key mechanism driving $\mathrm{T}$ cell exhaustion and immune tolerance in the tumor microenvironment. CTLA-4 and PD-1 are well recognized immune checkpoints in cancer expressed on activated $\mathrm{T}$ cells and other immune cells. CTLA-4 is also expressed on T regulatory cells (Tregs) and upon antigen presentation exerts its inhibitory effect by counteracting $\mathrm{T}$ cell co stimulation. This is achieved via competitive binding of CD80 and CD86 receptors on antigen presenting cells leading to diminished CD28 activation and immune evasion (33-37). Downregulation of the CD28 pathway is also affected by PD-1 binding to its ligands, PD-L1 and PD-L2 inhibiting CD8 ${ }^{+} \mathrm{T}$ cell activation and leading to immune suppression (Figure 3). Cancer cells utilize this mechanism to evade immune surveillance by expressing PD-L1 and PD-L2. A state of $\mathrm{T}$ cell exhaustion is reached through chronic antigen exposure in the tumor microenvironment evident by upregulation of PD-L1 in antigen presenting cells and tumor cells induced by PD-1 expression on reactive T cells $(34,38,39)$. This leads to tumor infiltration, less efficient tumor control and as a result poorer outcome (40).

Over the last 5-10 years we have appreciated the evolution of immunotherapy and its positive outcomes in the treatments of multiple cancers including melanoma, non-small cell lung cancer, renal cell carcinoma, head and neck cancers, Hodgkin's disease, and others. In HCC tumor antigen therapy, immune checkpoint inhibitors, and adoptive cell transfer (ACT) have all been investigated (41). The potential use of immunotherapy in HCC was first investigated with tremelimumab, a cytotoxic $\mathrm{T}$ lymphocyte antigen 4 (CTLA4) human monoclonal IgG2 antibody. This multicenter phase II trial evaluated 21 patients with HCC and chronic hepatitis $\mathrm{C}$ who were treated with tremelimumab (42). A good safety profile was observed and a subsequent median TTP of 6.5 months (95\% CI, 3.95-9.14), and median OS of 8.2 months (95\% CI, 4.64-21.34) were observed. Accelerated FDA approval was obtained for nivolumab [an IgG4 monoclonal antibody targeting programmed cell death 1 (PD-1)] in the treatment of HCC in patients previously treated with sorafenib. This came after the milestone CheckMate-040 trial, which evaluated nivolumab monotherapy, reported $70 \%$ of patients previously treated with sorafenib achieved an ORR of $20 \%$ in the dose-escalation cohort and $15 \%$ in the dose-expansion cohort [with complete responses (CR) achieved in each group]; stable disease was shown in $43.2 \%$ and $45.5 \%$, respectively. Efficacy was seen irrespective of PD-1 expression on tumor cells, hepatitis B or C viral infection (43). This led to the phase III CheckMate-459 study which evaluated nivolumab in the first-line setting but did not meet its primary endpoint of OS even though showing clinically meaningful improvements in OS, ORR, and CR (44) (Table 3). Pembrolizumab, another anti-PD1 monoclonal antibody, also demonstrated clinical antitumor activity and safety in the phase II KEYNOTE-224 trial in patients previously treated with sorafenib in advanced HCC. The phase III KEYNOTE 240 randomized trial evaluated pembrolizumab and showed improved OS compared with placebo $(\mathrm{HR}=0.78$; one-sided $\mathrm{P}=0.0238)$ and PFS (HR $=0.78$; one-sided $\mathrm{P}=0.0209$ ) but failed to reach statistical significance (46).

Chimeric antigen receptor $\mathrm{T}$ cells (CAR-T), a form of ACT therapy, has shown promising results in the treatment of $\mathrm{CD} 19^{+}$hematological malignancies. Its utility in solid tumors, and specifically HCC, has unfortunately 
A

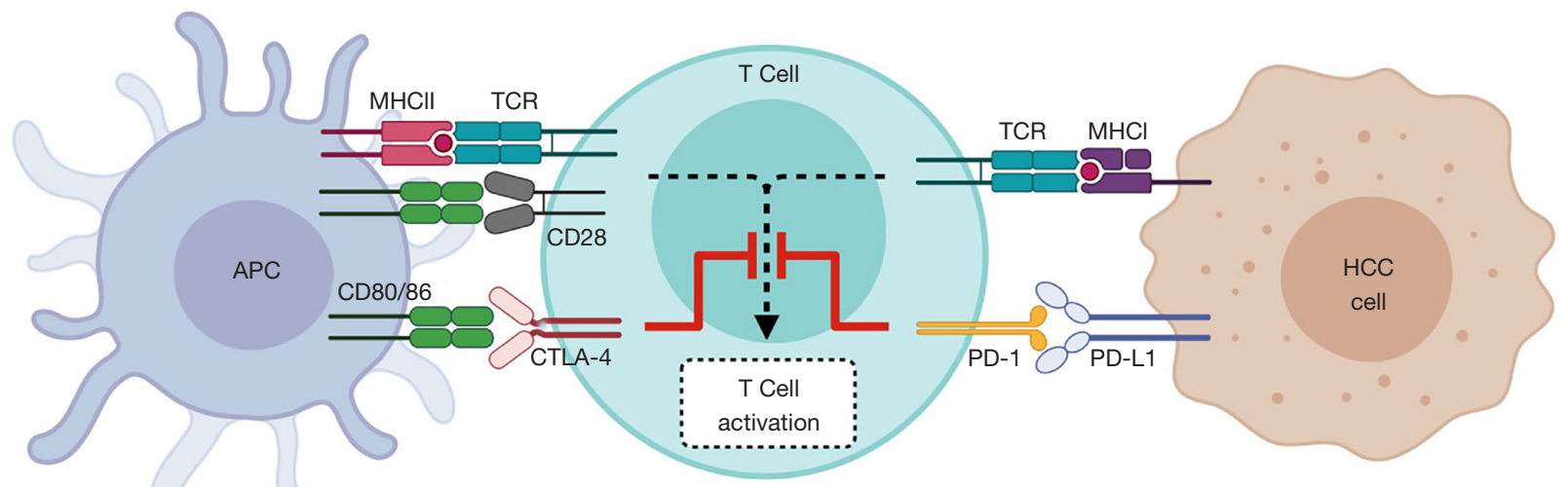

B

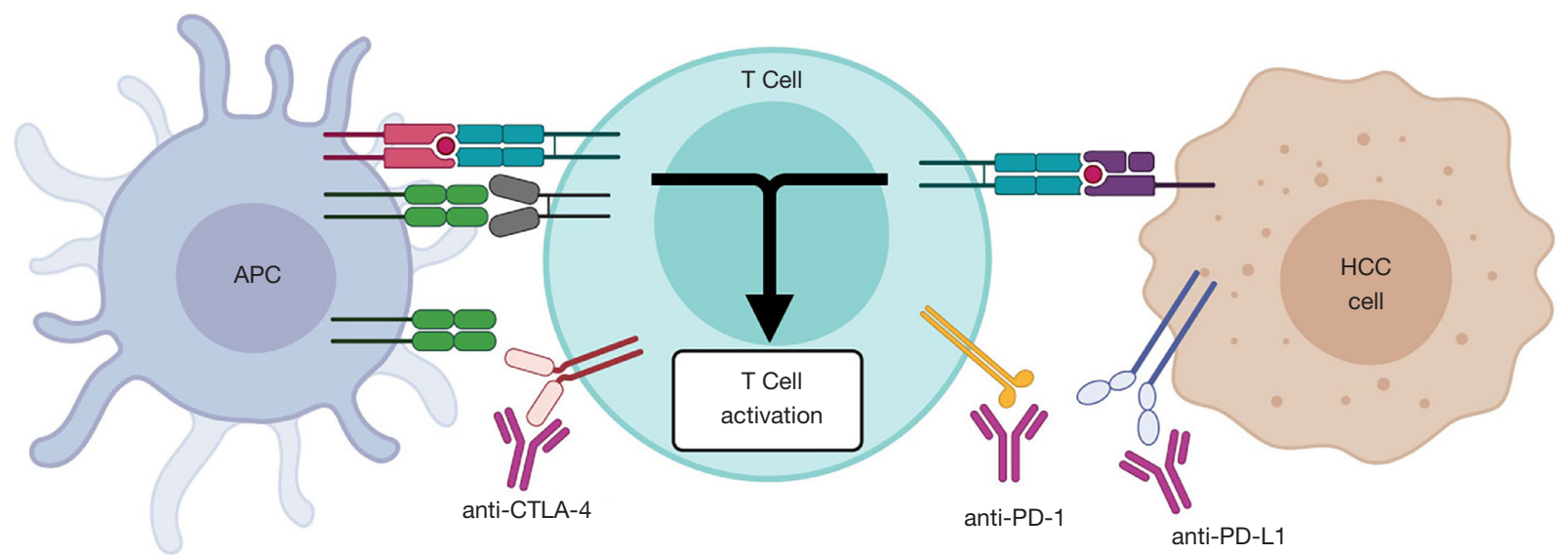

Figure 3 Mechanism of immune checkpoints in HCC in absence (A) and presence (B) of ICI. The figure is reused from the article below Mohr R, Jost-Brinkmann F, Özdirik B, et al. Lessons From Immune Checkpoint Inhibitor Trials in Hepatocellular Carcinoma. Front Immunol 2021;12:652172. APC, antigen presenting cell; CTLA-4, cytotoxic T-lymphocyte associated protein 4; HCC, hepatocellular carcinoma; ICI, immune checkpoint inhibitor; MHC, major histocompatibility complex; PD-1, programmed cell death 1 protein; PD-L1, programmed death-ligand 1; TCR, T cell receptor.

not been as promising; either due to a lack of efficacy or due to intolerable toxicities. The concept behind CAR-T immunotherapy is that tumor cells express antigens or aberrant proteins that can be targeted with genetically engineered $\mathrm{T}$ cells $(41,47)$.

Preclinical data in HCC tumors have shown promising results with various tumor associated antigens that could be potential targets for CAR-T therapy, specifically GPC3, MUC-1, EpCAM, CEA and AFP (47). Future directions would be to translate some of these potential outcomes into clinical trials.

\section{Combination therapy}

The most recent and remarkable advance in HCC has been with combination therapy atezolizumab, an anti PD-L1 antibody, and bevacizumab, a VEGF monoclonal antibody for unresectable or metastatic HCC (48). VEGF overexpression has been evident in the tumor microenvironment contributing to immune evasion and tolerance in tumors. VEGF is primarily involved in angiogenesis which paradoxically leads to a hypoxic and acidotic TME associated with recruitment of immunosuppressive cells such as Tregs (49). VEGF also increases PD-1 expression of tumor infiltrating T-cells (50). VEGF mediated immune suppression is reduced by targeting VEGF and the addition of immune checkpoint inhibitors results in an enhanced immune reactivation via upregulation of $\mathrm{T}$ cell activity and infiltration into tumor cells (48). This combination was evaluated in the phase 
Table 3 Single agent immunotherapy trials in HCC

\begin{tabular}{|c|c|c|c|c|c|c|}
\hline Trial & Author & $\begin{array}{l}\text { No. of } \\
\text { patients }\end{array}$ & Study arm & ECOG & $\begin{array}{l}\text { Child-Pugh } \\
\text { score }\end{array}$ & Primary end point \\
\hline & $\begin{array}{l}\text { Sangro } \\
\text { et al. (42) }\end{array}$ & 21 & $\begin{array}{l}\text { Tremelimumab in } \\
\text { chronic hepatitis C }\end{array}$ & 0 or 1 & $A$ or $B$ & $\begin{array}{l}\text { Safety and efficacy: TTP was } 6.48 \text { months }(95 \% \mathrm{Cl} \text {, } \\
\text { 3.95-9.14) and median OS was } 8.2 \text { months }(95 \% \mathrm{Cl} \text {, } \\
\text { 4.64-21.34) }\end{array}$ \\
\hline & $\begin{array}{l}\text { Wainberg } \\
\text { et al. (45) }\end{array}$ & 40 & $\begin{array}{l}\text { Durvalumab } \\
\text { (phase I/II) }\end{array}$ & 0 or 1 & A & $\begin{array}{l}\text { Safety and efficacy: median OS } 13.2 \text { months (6.3-21.1); } \\
\text { ORR } 10.3 \%(2.9-24.2)\end{array}$ \\
\hline Keynote-240 & $\begin{array}{l}\text { Finn } \\
\text { et al. (46) }\end{array}$ & 413 & $\begin{array}{l}\text { Pembrolizumab vs. } \\
\text { placebo (phase III, } \\
\text { second-line setting) }\end{array}$ & 0 or 1 & A & $\begin{array}{l}\text { OS: } 13.9 \text { months }(95 \% \mathrm{Cl}, 11.6-16.0) \text { for pembro vs. } \\
10.6 \text { months }(95 \% \mathrm{Cl}, 8.3-13.5) \text { for placebo ( } \mathrm{HR}=0.781 \text {; } \\
95 \% \mathrm{Cl}, 0.611-0.998 ; \mathrm{P}=0.0238) \text {; PFS: } 3.0 \text { months }(95 \% \\
\mathrm{Cl}, 2.8-4.1) \text { vs. } 2.8 \text { months }(95 \% \mathrm{Cl}, 1.6-3.0)\end{array}$ \\
\hline CheckMate-459 & $\begin{array}{l}\text { Yau } \\
\text { et al. (44) }\end{array}$ & 743 & $\begin{array}{l}\text { Nivolumab vs. } \\
\text { sorafenib (phase III, } \\
\text { first-line setting) }\end{array}$ & & & $\begin{array}{l}\text { OS: } 16.4 \text { months for nivolumab vs. } 14.7 \text { months for } \\
\text { sorafenib (HR }=0.85 ; 95 \% \mathrm{Cl}, 0.72-1.02 ; \mathrm{P}=0.0752) \text {; } \\
\text { ORR: } 15 \% \text { with nivolumab ( } 14 \text { patients with } \mathrm{CR}) \text { vs. } 7 \% \\
\text { with sorafenib ( } 5 \text { patients with } \mathrm{CR} \text { ) }\end{array}$ \\
\hline
\end{tabular}

HCC, hepatocellular carcinoma; ORR, objective response rate; OS, overall survival; HR, hazard ratio; CR, complete response.

III IMbrave150 trial that randomized 501 patients who had not received prior systemic treatment in a 2:1 ratio to atezolizumab-bevacizumab vs. sorafenib. The study showed statistically significant improvement in OS at 12 months was $67.2 \%$ (95\% CI, 61.3-73.1\%) with atezolizumabbevacizumab and $54.6 \%$ (95\% CI, 45.2-64.0\%) with sorafenib (51). PFS HR was 6.8 months compared with 4.3 months with sorafenib 0.59 (95\% CI, 0.47-0.76; $\mathrm{P}<0.0001)$ (48). Secondary endpoints further demonstrated ORR of $27.3 \%$ (95\% CI, 22.5-32.5\%) with atezolizumabbevacizumab and $11.9 \%$ (95\% CI, 7.4-18.0\%) with sorafenib according to RECIST 1.1. This promising breakthrough has led to this combination becoming the preferred approach for HCC management with FDA approval and is now recommended as first-line therapy in advanced HCC per the 2020 ASCO guidelines (52). The adverse event profile of this combination was consistent with the known safety profile of each agent and the underlying disease. Grade 3-4 hypertension was observed in $15.2 \%$ of patients and less commonly proteinuria, fatigue, elevation in AST levels were also reported. Approximately $15 \%$ of patients in the atezolizumab-bevacizumab group discontinued treatment based on adverse events compared to $10 \%$ in the sorafenib group. Gastrointestinal adverse events were the most common reported reason for discontinuation. Bleeding is a known adverse event of bevacizumab attributed to its anti-angiogenic effects. Upper gastrointestinal bleeding with the potential for a lifethreatening bleed is a common complication of cirrhosis and in the atezolizumab-bevacizumab group this was observed in $7 \%$ of patients compared to $4.5 \%$ on sorafenib (51). The major caveat to note is that patients must have no esophagogastric varices (or varices should have been adequately treated prior to treatment initiation) due to increased risk of catastrophic bleeding with bevacizumab.

Further research into potential combination therapies continue to primarily involve immunomodulation by incorporating ICI's with either TKI's, alternative ICI/ anti-VEGF combinations or dual immune checkpoint inhibition. Preclinical data into combination ICI and TKI's revealed the immunomodulatory effects of TKI's, specifically lenvatinib shown to reduce tumor-associated macrophages (TAMs) and increased the percentage of activated $\mathrm{CD} 8^{+} \mathrm{T}$ cells secreting interferon (IFN) $\gamma(53)$. When combined with PD-1 blockade anti-tumor activity was enhanced via the IFN signaling pathway (53). The phase Ib KEYNOTE-524 identifies pembrolizumab and lenvatinib as a first-line treatment option in advanced HCC. The latest data from 2020 ASCO meeting showed median OS was 22.0 months (95\% CI, 20.4-NE), the median PFS was 9.3 months (95\% CI, 7.1-9.7), and the ORR was $46 \%$ (95\% CI, 36-56.3\%), CR reaches 11\% (54). Grade $\geq 3$ treatment related adverse effects occurred in $67 \%$ of patients consistent with the known toxicities of each drug. This promising therapeutic combination has launched the LEAP-002 global phase III evaluating pembrolizumablenvatinib against lenvatinib monotherapy (55). Recently published data evaluated combination TKI/anti-PD1 
therapy as a downstaging strategy to convert previously unresectable HCC to resectable HCC. Treatment utilized either pembrolizumab-lenvatinib and camrelizumab-apatinib in 63 patients and $15 \%$ of these patients were able to receive curative resection (56). Camrelizumab, an anti-PD-1 and apatinib, an anti-VEGF-2 TKI was further evaluated in the RESCUE trial. This phase II study enrolled 190 patients with advanced HCC who were either treatment naïve or refractory/intolerant to $1^{\text {st }}$ or $2^{\text {nd }}$ line therapy. The ORR was $34.3 \%$ (95\% CI, 23.3-46.6\%) in the first-line cohort and $22.5 \%$ (95\% CI, 15.4-31.0\%) in the second-line cohort. Median PFS in both cohorts was 5.7 months (95\% CI, 5.47.4 ) and 5.5 months (95\% CI, 3.7-5.6) respectively. Survival rate at 12 -months was $74.7 \%$ (95\% CI, $62.5-83.5 \%)$ in the $1^{\text {st }}$ line cohort and $68.2 \%$ (95\% CI, $59.0-75.7 \%$ ) in those with prior $2^{\text {nd }}$ line therapy. A proportion of $77 \%$ of patients experienced grade $\geq 3$ therapy related adverse events the most common being hypertension (57). This promising outcome has translated to the phase III trial currently active (NCT03764293). The phase Ib VEGF Liver 100 trial evaluated avelumab, an anti-PD-L1 antibody with axitinib, a selective TKI to VEGF 1, 2, 3. Overall this combination was well tolerated with reported adverse effects consistent with each drug such as hypertension, hand-foot skin reactions, hypo- and hyperthyroidism. No grade $4 / 5$ treatment related adverse events were reported. In the 22 patients that were evaluated tumor shrinkage was observed in $15(68.2 \%)$ and $16(72.7 \%)$ patients, ORR was $13.6 \%$ (95\% CI, $2.9-34.9 \%$ ) and $31.8 \%$ (95\% CI, $13.9-54.9 \%$ ) by RECIST and mRECIST, respectively (58).

\section{Conclusions}

Over the past 15 years, we have made remarkable progress in the management of advanced HCC-from sorafenib dominating as the sole option in the first-line setting to now having three different first-line treatment options (atezolizumab-bevacizumab, sorafenib and lenvatinib). Second-line treatment options have also expanded to cabozantinib, regorafenib, ramucirumab (AFP $>400 \mathrm{ng} / \mathrm{mL}$ ). The immune checkpoint inhibitors nivolumab and pembrolizumab have been recommended as second-line treatment in patients intolerant to TKI's (50). With the success of combination azetolizumab-bevacizumab, ongoing studies are currently looking into more combination therapies with ICI's as the backbone. This includes pembrolizumab-lenvatinib in the LEAP-002 phase III (55), atezolizumab-cabozantinib in the COSMIC-012 phase
III (59), and doublet immune checkpoint inhibition with nivolumab-ipilimumab in the Checkmate-9DW trial (60) and durvalumab-tremelimumab in the HIMALAYA phase III trial (61). It is with great expectation that as research continues into investigating various targeted therapeutic options in HCC, more therapies will become available that translate into longer survival benefits.

\section{Acknowledgments}

Funding: None.

\section{Footnote}

Reporting Checklist: The authors have completed the Narrative Review reporting checklist. Available at https:// jgo.amegroups.com/article/view/10.21037/jgo-21-274/rc

Conflicts of Interest: Both authors have completed the ICMJE uniform disclosure form (available at https://jgo. amegroups.com/article/view/10.21037/jgo-21-274/coif). DS reports institutional affiliations with Amgen, Apixigen, BMS, Fibrogen, Rafael, Oncomed, Merca, Roche with no personal fees and affiliations with Incyte and Genetech associated with personal fees. JA has no conflicts of interest to declare.

Ethical Statement: The authors are accountable for all aspects of the work in ensuring that questions related to the accuracy or integrity of any part of the work are appropriately investigated and resolved.

Open Access Statement: This is an Open Access article distributed in accordance with the Creative Commons Attribution-NonCommercial-NoDerivs 4.0 International License (CC BY-NC-ND 4.0), which permits the noncommercial replication and distribution of the article with the strict proviso that no changes or edits are made and the original work is properly cited (including links to both the formal publication through the relevant DOI and the license). See: https://creativecommons.org/licenses/by-nc-nd/4.0/.

\section{References}

1. Ferlay J, Shin HR, Bray F, et al. Estimates of worldwide burden of cancer in 2008: GLOBOCAN 2008. Int J Cancer 2010;127:2893-917.

2. Global Burden of Disease Liver Cancer Collaboration; 
Akinyemiju T, Abera S, et al. The Burden of Primary Liver Cancer and Underlying Etiologies From 1990 to 2015 at the Global, Regional, and National Level: Results From the Global Burden of Disease Study 2015. JAMA Oncol 2017;3:1683-91.

3. Rahib L, Wehner MR, Matrisian LM, et al. Estimated Projection of US Cancer Incidence and Death to 2040. JAMA Netw Open 2021;4:e214708.

4. Balogh J, Victor D 3rd, Asham EH, et al. Hepatocellular carcinoma: a review. J Hepatocell Carcinoma 2016;3:41-53.

5. Llovet JM, Ricci S, Mazzaferro V, et al. Sorafenib in advanced hepatocellular carcinoma. $\mathrm{N}$ Engl J Med 2008;359:378-90.

6. Llovet JM, Zucman-Rossi J, Pikarsky E, et al. Hepatocellular carcinoma. Nat Rev Dis Primers 2016;2:16018.

7. Jiao Q, Bi L, Ren Y, et al. Advances in studies of tyrosine kinase inhibitors and their acquired resistance. Mol Cancer 2018;17:36.

8. Kudo M, Finn RS, Qin S, et al. Lenvatinib versus sorafenib in first-line treatment of patients with unresectable hepatocellular carcinoma: a randomised phase 3 noninferiority trial. Lancet 2018;391:1163-73.

9. Abou-Alfa GK, Meyer T, Cheng AL, et al. Cabozantinib in Patients with Advanced and Progressing Hepatocellular Carcinoma. N Engl J Med 2018;379:54-63.

10. Bruix J, Qin S, Merle P, et al. Regorafenib for patients with hepatocellular carcinoma who progressed on sorafenib treatment (RESORCE): a randomised, double-blind, placebo-controlled, phase 3 trial. Lancet 2017;389:56-66.

11. Cheng A, Kang Y, Lin D, et al. Phase III trial of sunitinib $(\mathrm{Su})$ versus sorafenib $(\mathrm{So})$ in advanced hepatocellular carcinoma (HCC). J Clin Oncol 2011;29:4000.

12. Johnson PJ, Qin S, Park JW, et al. Brivanib versus sorafenib as first-line therapy in patients with unresectable, advanced hepatocellular carcinoma: results from the randomized phase III BRISK-FL study. J Clin Oncol 2013;31:3517-24.

13. Cainap C, Qin S, Huang WT, et al. Linifanib versus Sorafenib in patients with advanced hepatocellular carcinoma: results of a randomized phase III trial. J Clin Oncol 2015;33:172-9.

14. Zhu AX, Rosmorduc O, Evans TR, et al. A phase III, randomized, double-blind, placebo-controlled trial of sorafenib plus erlotinib in patients with advanced hepatocellular carcinoma. J Clin Oncol 2015;33:559-66.

15. Cersosimo RJ. Systemic targeted and immunotherapy for advanced hepatocellular carcinoma. Am J Health Syst Pharm 2021;78:187-202.
16. Sullivan LA, Brekken RA. The VEGF family in cancer and antibody-based strategies for their inhibition. MAbs 2010;2:165-75.

17. Zhu AX, Park JO, Ryoo BY, et al. Ramucirumab versus placebo as second-line treatment in patients with advanced hepatocellular carcinoma following first-line therapy with sorafenib (REACH): a randomised, double-blind, multicentre, phase 3 trial. Lancet Oncol 2015;16:859-70.

18. Folkman J. Tumor angiogenesis: therapeutic implications. N Engl J Med 1971;285:1182-6.

19. Fang P, Hu JH, Cheng ZG, et al. Efficacy and safety of bevacizumab for the treatment of advanced hepatocellular carcinoma: a systematic review of phase II trials. PLoS One 2012;7:e49717.

20. Huang J, Zhang X, Tang Q, et al. Prognostic significance and potential therapeutic target of VEGFR2 in hepatocellular carcinoma. J Clin Pathol 2011;64:343-8.

21. Zhu AX, Kang YK, Yen CJ, et al. REACH-2: A randomized, double-blind, placebo-controlled phase 3 study of ramucirumab versus placebo as secondline treatment in patients with advanced hepatocellular carcinoma (HCC) and elevated baseline alpha-fetoprotein (AFP) following first-line sorafenib. J Clin Oncol 2018;36:4003.

22. Siegel AB, Cohen EI, Ocean A, et al. Phase II trial evaluating the clinical and biologic effects of bevacizumab in unresectable hepatocellular carcinoma. J Clin Oncol 2008;26:2992-8.

23. Kaseb AO, Garrett-Mayer E, Morris JS, et al. Efficacy of bevacizumab plus erlotinib for advanced hepatocellular carcinoma and predictors of outcome: final results of a phase II trial. Oncology 2012;82:67-74.

24. Thomas MB, Morris JS, Chadha R, et al. Phase II trial of the combination of bevacizumab and erlotinib in patients who have advanced hepatocellular carcinoma. J Clin Oncol 2009;27:843-50.

25. Yau T, Wong H, Chan P, et al. Phase II study of bevacizumab and erlotinib in the treatment of advanced hepatocellular carcinoma patients with sorafenib-refractory disease. Invest New Drugs 2012;30:2384-90.

26. Philip PA, Mahoney MR, Holen KD, et al. Phase 2 study of bevacizumab plus erlotinib in patients with advanced hepatocellular cancer. Cancer 2012;118:2424-30.

27. Hsu CH, Yang TS, Hsu C, et al. Efficacy and tolerability of bevacizumab plus capecitabine as first-line therapy in patients with advanced hepatocellular carcinoma. $\mathrm{Br} \mathrm{J}$ Cancer 2010;102:981-6.

28. Sun W, Sohal D, Haller DG, et al. Phase 2 trial of 
bevacizumab, capecitabine, and oxaliplatin in treatment of advanced hepatocellular carcinoma. Cancer 2011;117:3187-92.

29. Zhu AX, Blaszkowsky LS, Ryan DP, et al. Phase II study of gemcitabine and oxaliplatin in combination with bevacizumab in patients with advanced hepatocellular carcinoma. J Clin Oncol 2006;24:1898-903.

30. Leonardi GC, Candido S, Cervello M, et al. The tumor microenvironment in hepatocellular carcinoma (review). Int J Oncol 2012;40:1733-47.

31. Prieto J, Melero I, Sangro B. Immunological landscape and immunotherapy of hepatocellular carcinoma. Nat Rev Gastroenterol Hepatol 2015;12:681-700.

32. Szturz P, Vermorken JB. Further clinical interpretation and implications of KEYNOTE-048 findings. Lancet 2020;396:379.

33. Mohr R, Jost-Brinkmann F, Özdirik B, et al. Lessons From Immune Checkpoint Inhibitor Trials in Hepatocellular Carcinoma. Front Immunol 2021;12:652172.

34. Iñarrairaegui M, Melero I, Sangro B. Immunotherapy of Hepatocellular Carcinoma: Facts and Hopes. Clin Cancer Res 2018;24:1518-24.

35. Wing K, Onishi Y, Prieto-Martin P, et al. CTLA-4 control over Foxp3 + regulatory T cell function. Science 2008;322:271-5.

36. Han Y, Chen Z, Yang Y, et al. Human CD14+ CTLA4+ regulatory dendritic cells suppress $T$-cell response by cytotoxic T-lymphocyte antigen-4-dependent IL-10 and indoleamine-2,3-dioxygenase production in hepatocellular carcinoma. Hepatology 2014;59:567-79.

37. Cariani E, Missale G. Immune landscape of hepatocellular carcinoma microenvironment: Implications for prognosis and therapeutic applications. Liver Int 2019;39:1608-21.

38. Pardoll DM. The blockade of immune checkpoints in cancer immunotherapy. Nat Rev Cancer 2012;12:252-64.

39. Barber DL, Wherry EJ, Masopust D, et al. Restoring function in exhausted CD8 $\mathrm{T}$ cells during chronic viral infection. Nature 2006;439:682-7.

40. Ni L, Feng Y, Dong C. The advancement of immunotherapy in hepatocellular carcinoma. Hepatoma Res 2020;6:25.

41. Medavaram S, Zhang Y. Emerging therapies in advanced hepatocellular carcinoma. Exp Hematol Oncol 2018;7:17.

42. Sangro B, Gomez-Martin C, de la Mata M, et al. A clinical trial of CTLA-4 blockade with tremelimumab in patients with hepatocellular carcinoma and chronic hepatitis C. J Hepatol 2013;59:81-8.

43. El-Khoueiry AB, Sangro B, Yau T, et al. Nivolumab in patients with advanced HCC (CheckMate 040): an openlabel, non-comparative, phase 1/2 dose escalation and expansion trial. Lancet 2017;389:2492-502.

44. Yau T, Park JW, Finn RS, et al. LBA38_PR - CheckMate 459: A randomized, multi-center phase III study of nivolumab (NIVO) vs sorafenib (SOR) as first-line (1L) treatment in patients (pts) with advanced hepatocellular carcinoma (aHCC). J Clin Oncol 2019;30:v874-5.

45. Wainberg ZA, Segal NH, Jaeger D, et al. Safety and clinical activity of durvalumab monotherapy in patients with hepatocellular carcinoma (HCC). J Clin Oncol 2017;35:4071.

46. Finn RS, Ryoo BY, Merle P, et al. Pembrolizumab As Second-Line Therapy in Patients With Advanced Hepatocellular Carcinoma in KEYNOTE-240: A Randomized, Double-Blind, Phase III Trial. J Clin Oncol 2020;38:193-202.

47. Liu B, Song Y, Liu D. Clinical trials of CAR-T cells in China. J Hematol Oncol 2017;10:166.

48. Finn RS, Qin S, Ikeda M, et al. Atezolizumab plus Bevacizumab in Unresectable Hepatocellular Carcinoma. N Engl J Med 2020;382:1894-905.

49. Dong Y, Wong JSL, Sugimura R, et al. Recent Advances and Future Prospects in Immune Checkpoint (ICI)-Based Combination Therapy for Advanced HCC. Cancers (Basel) 2021;13:1949.

50. Ramjiawan RR, Griffioen AW, Duda DG. Antiangiogenesis for cancer revisited: Is there a role for combinations with immunotherapy? Angiogenesis 2017;20:185-204.

51. Bomze D, Meirson T, Azoulay D. Atezolizumab and Bevacizumab in Hepatocellular Carcinoma. N Engl J Med 2020;383:693-4.

52. Gordan JD, Kennedy EB, Abou-Alfa GK, et al. Systemic Therapy for Advanced Hepatocellular Carcinoma: ASCO Guideline. J Clin Oncol 2020;38:4317-45.

53. Kato Y, Tabata K, Kimura T, et al. Lenvatinib plus antiPD-1 antibody combination treatment activates CD8+ T cells through reduction of tumor-associated macrophage and activation of the interferon pathway. PLoS One 2019; 14:e0212513.

54. Finn RS, Ikeda M, Zhu AX, et al. Phase Ib Study of Lenvatinib Plus Pembrolizumab in Patients With Unresectable Hepatocellular Carcinoma. J Clin Oncol 2020;38:2960-70.

55. Llovet JM, Kudo M, Cheng A, et al. Lenvatinib (len) plus pembrolizumab (pembro) for the first-line treatment of patients (pts) with advanced hepatocellular carcinoma 
(HCC): Phase 3 LEAP-002 study. J Clin Oncol 2019;37:4152.

56. Zhu XD, Huang C, Shen YH, et al. Downstaging and Resection of Initially Unresectable Hepatocellular Carcinoma with Tyrosine Kinase Inhibitor and Anti-PD-1 Antibody Combinations. Liver Cancer 2021;10:320-9.

57. Xu J, Shen J, Gu S, et al. Camrelizumab in Combination with Apatinib in Patients with Advanced Hepatocellular Carcinoma (RESCUE): A Nonrandomized, Open-label, Phase II Trial. Clin Cancer Res 2021;27:1003-11.

58. Kudo M, Motomura K, Wada Y, et al. First-line avelumab + axitinib in patients with advanced hepatocellular carcinoma: Results from a phase $1 \mathrm{~b}$ trial (VEGF Liver 100). J Clin Oncol 2019;37:4072.

Cite this article as: Awosika J, Sohal D. A narrative review of systemic treatment options for hepatocellular carcinoma: state of the art review. J Gastrointest Oncol 2022;13(1):426-437. doi: 10.21037/jgo-21-274
59. Kelley RK, W Oliver J, Hazra S, et al. Cabozantinib in combination with atezolizumab versus sorafenib in treatment-naive advanced hepatocellular carcinoma: COSMIC-312 Phase III study design. Future Oncol 2020;16:1525-36.

60. A study of nivolumab in combination with ipilimumab in participants with advanced hepatocellular carcinoma (CheckMate 9DW). Available online: https://clinicaltrials. gov/ct2/show/NCT04039607

61. Abou-Alfa GK, Chan SL, Furuse J, et al. A randomized, multicenter phase 3 study of durvalumab (D) and tremelimumab $(\mathrm{T})$ as first-line treatment in patients with unresectable hepatocellular carcinoma (HCC): HIMALAYA study. J Clin Oncol 2018;36:TPS4144. 